\title{
Minimum Quality Threshold in Pre-Clinical Sepsis Studies (MQTiPSS): an international expert consensus initiative for improvement of animal modeling in sepsis
}

\author{
Marcin F. Osuchowski ${ }^{1}$ - Alfred Ayala ${ }^{2}$. Soheyl Bahrami ${ }^{1} \cdot$ Michael Bauer $^{3} \cdot$ Mihaly Boros $^{4} \cdot$ Jean-Marc Cavaillon ${ }^{5}$. \\ Irshad H. Chaudry ${ }^{6}$. Craig M. Coopersmith ${ }^{7}$. Clifford Deutschman ${ }^{8}$. Susanne Drechsler ${ }^{1} \cdot$ Philip Efron $^{9}$. \\ Claes Frostell ${ }^{10}$. Gerhard Fritsch ${ }^{11,12}$. Waldemar Gozdzik ${ }^{13}$. Judith Hellman ${ }^{14}$. Markus Huber-Lang ${ }^{15}$. \\ Shigeaki Inoue ${ }^{16}$. Sylvia Knapp ${ }^{17}$. Andrey V. Kozlov ${ }^{1}$. Claude Libert ${ }^{18,19}$. John C. Marshall ${ }^{20}$. Lyle L. Moldawer $^{9}$. \\ Peter Radermacher ${ }^{21}$. Heinz Redl ${ }^{1}$. Daniel G. Remick ${ }^{22}$ - Mervyn Singer ${ }^{23}$. Christoph Thiemermann ${ }^{24} \cdot$ Ping Wang $^{25}$. \\ W. Joost Wiersinga ${ }^{26} \cdot$ Xianzhong Xiao $^{27} \cdot$ Basilia Zingarelli $^{28}$
}

Published online: 14 August 2018

(c) The Author(s) 2018, corrected publication August 2018

\begin{abstract}
Purpose Pre-clinical animal studies precede the majority of clinical trials. While the clinical sepsis definitions and recommended treatments are regularly updated, a systematic review of pre-clinical models of sepsis has not been done and clear modeling guidelines are lacking. To address this deficit, a Wiggers-Bernard Conference on pre-clinical sepsis modeling was held in Vienna in May, 2017. The conference goal was to identify limitations of pre-clinical sepsis models and to propose a set of guidelines, defined as the "Minimum Quality Threshold in Pre-Clinical Sepsis Studies" (MQTiPSS), to enhance translational value of these models.

Methods 31 experts from 13 countries participated and were divided into 6 thematic Working Groups (WG): (1) Study Design, (2) Humane modeling, (3) Infection types, (4) Organ failure/dysfunction, (5) Fluid resuscitation and (6) Antimicrobial therapy endpoints. As basis for the MQTiPSS discussions, the participants conducted a literature review of the 260 most highly cited scientific articles on sepsis models (2002-2013).

Results Overall, the participants reached consensus on 29 points; 20 at "recommendation" (R) and 9 at "consideration" (C) strength. This Executive Summary provides a synopsis of the MQTiPSS consensus (Tables 1, 2 and 3).

Conclusions We believe that these recommendations and considerations will serve to bring a level of standardization to pre-clinical models of sepsis and ultimately improve translation of pre-clinical findings. These guideline points are proposed as "best practices" that should be implemented for animal sepsis models. In order to encourage its wide dissemination, this article is freely accessible in Shock, Infection and Intensive Care Medicine Experimental.
\end{abstract}

Keywords Guidelines · Experiment · Study design · Humane modeling · Infection types · Organ dysfunction · Fluid resuscitation · Antimicrobial therapy

“This modeling thing, it's pretty easy, but actually it's also really tough." Cara Delevingne

Marcin F. Osuchowski

marcin.osuchowski@trauma.lbg.ac.at

Extended author information available on the last page of the article

\section{The necessity}

With the ultimate goal to reduce mortality/morbidity in patients, animal modeling of diseases has been limited by poor translation $[1,2]$. This is often fueled by the low fidelity of available model systems [3, 4], their inappropriate study designs [2] and selective use of animal data [5, 6]. 
Table 1 Combined Recommendations and Considerations from the Working Group (WG) 1 and 2

Study Design

(WG-1)

1. Survival follow-up should reasonably reflect the clinical time course of the sepsis model

2. Therapeutic interventions should be initiated after the septic insult replicating clinical care

3. We recommend that the treatment be randomized and blinded when feasible

4. Provide as much information as possible (e.g. ARRIVE guidelines) on the model and methodology, to enable replication.

a. Consider replication of the findings in models that include co-morbidity and/or other biological variables (i.e., age, gender, diabetes, cancer, immuno-suppression, genetic background and others).

b. In addition to rodents (mice and rats), consider modeling sepsis also in other (mammal) species.

c. Consider need for source control

Humane Modeling (WG-2)
5. The development and validation of standardized criteria to monitor the well-being of septic animals is recommended

6. The development and validation of standardized criteria for euthanasia of septic animals is recommended (exceptions possible)

7. Analgesics recommended for surgical sepsis consistent with ethical considerations

d. Consider analgesics for nonsurgical sepsis

R: Recommendation strength; C: consideration strength

Table 2 Combined Recommendations and Considerations from the Working Group (WG) 3 and 4

\begin{tabular}{ll}
\hline Infection Types & 8. We recommend that challenge with LPS is not an appropriate model for replicating human sepsis \\
(WG-3) & 9. We recommend that microorganisms used in animal models preferentially replicate those commonly found \\
in human sepsis \\
$\begin{array}{l}\text { e. Consider modeling sepsis syndromes that are initiated at sites other than the peritoneal cavity (e.g. lung, } \\
\text { urinary tract, brain) }\end{array}$
\end{tabular}

Organ Failure/ Dysfunction (WG-4)

10. Organ/system dysfunction is defined as life threatening deviation from normal for that organ/system based on objective evidence

11. Not all activities in an individual organ/system need to be abnormal for organ dysfunction to be present

12. To define objective evidence of the severity of organ/system dysfunction, a scoring system should be developed, validated and used, or use an existing scoring system.

13. Not all experiments must measure all parameters of organ dysfunction but animal models should be fully exploited

f. Avoid hypoglycemia

$\mathrm{R}$ : Recommendation strength; C: consideration strength

Table 3 Combined Recommendations and Considerations from the Working Group (WG) 5 and 6

\section{Fluid Resuscitation}

(WG-5)

14. Fluid resuscitation is essential unless part of the study

15. Administer fluid resuscitation based on the specific requirements of the model

16. Consider the specific sepsis model for the timing of the start and continuation for fluid resuscitation

17. Resuscitation is recommended by the application of iso-osmolar crystalloid solutions

g. Consider using pre-defined endpoints for fluid resuscitation as deemed necessary

h. Avoid fluid overload

\section{Anti-microbial Therapy (WG-6)}

18. Antimicrobials are recommended for pre-clinical studies assessing potential human therapeutics

19. Antimicrobials should be chosen based on the model and likely/known pathogen

20. Administration of antimicrobials should mimic clinical practice

i. Antimicrobials should be initiated after sepsis is established
$\mathbf{R}$ 
When compared to other inflammatory states (e.g. arthritis, atherosclerosis), the complexity of sepsis has hampered the development of high-fidelity models. However, this challenge can be aptly embraced by building on recent advances in the understanding of sepsis pathophysiology and avoiding past errors. Any promising sepsis model must be (a) specifically tailored to the posited hypothesis, (b) "reverse translated" to its clinical counterpart [7, 8] and (c) adjusted as new pathophysiological evidence emerges. This is echoed by the US Food and Drug Administration (FDA) in their 2010 Guidance for Industry and FDA Staff: "FDA believes that the animal...(model)... should provide a test system that offers a best attempt at simulating the clinical setting". (General Considerations for Animal Studies for Cardiovascular Devices; http://www.fda.gov).

Unfortunately, while the clinical definition of sepsis is currently in its third iteration [9] and the Surviving Sepsis Campaign (SSC) Guidelines for patient management have been updated three times [10], pre-clinical sepsis research has not been subjected to any organized attempt at introducing best practices, management guidelines and standardization [11]. This creates a large quality gap and confusion with conflicting data reflecting huge variations in, for example, insult severity, fluid resuscitation and study duration. Effective animal modeling and reporting guidelines have recently been proposed for other specific diseases such as pulmonary fibrosis [12], stroke [13, 14], heart failure [15] and malaria [16] making the void in the field of pre-clinical sepsis even more apparent. It is essential that animal models of sepsis continue to evolve. Lack of sufficient standardization of preclinical models will continue to limit the utility of sepsis animal research as a useful platform for advancing clinical outcomes and care in sepsis $[17,18]$ and will reduce the opportunities to identify and test new therapies.

\section{The action}

To address this perceived deficit, the Ludwig Boltzmann Institute of Experimental and Clinical Traumatology in the AUVA Research Center organized in May 2017 in Vienna a Wiggers-Bernard Conference on "Pre-clinical Modeling in Sepsis: Exchanging Opinions and Forming Recommendations". The key goal was to create publishable material that characterizes elements that should be included in pre-clinical sepsis studies and defined by the so called "Minimum Quality Threshold in Pre-Clinical Sepsis Studies" (MQTiPSS) descriptor. The Wiggers-Bernard Conference participants identified and addressed several broad, critically-important concepts in animal sepsis modeling. A total of 31 experts from 13 countries participated in the initiative (including five members of the Sepsis-3 definitions task force) and were divided into 6 thematic Working
Groups: (1) study design, (2) humane endpoints, (3) infection types, (4) organ failure/dysfunction, (5) critical fluid resuscitation and (6) antimicrobial therapy.

The initiative consisted of three phases: (a) preparatory (prior to the meeting; approximately 3 months), during which participants performed a systematic review of the 260 top cited (over 29,000 citations in aggregate) 2003-2012 pre-clinical publications (using ISI Web of Knowledge database; query: "sepsis model"; 374 individual experiments analyzed) and identified the key modeling topics to be discussed, (b) discussion during which the participants spent 2 days at the Wiggers-Bernard Conference examining pre-clinical sepsis models and ultimately voted to reach consensus on the proposed points (either at the "recommendation" or "consideration" strength), and (c) post-meeting refinement of the accepted points and finalization of the arguments to be included in the final publications (using a modified Delphi method; approximately 3 months). Following the format used by the Sepsis-3 task force [8], at least $2 / 3$ (over $65 \%$ ) of the votes were required for approval of a proposed point.

\section{The proposed outcome}

First, a definition for an animal model of sepsis was formulated and (unanimously) approved: "An experimental animal (mammal) model of sepsis should be defined as lifethreatening organ dysfunction caused by a dysregulated host response to an infection." Second, Wiggers-Bernard Conference participants reached consensus on 29 points; 20 at "recommendation" strength and 9 at "consideration" strength (listed in Tables 1, 2 and 3). All consensus points were reached either unanimously or with no more than 2 abstentions per point (point 8). The "recommendation" strength indicates virtually unanimous agreement among the 31 participants, regarding both the content and the need for rapid implementation. Issues that require additional discussion before final recommendations could be made were classified as considerations.

The current executive summary briefly describes the Wiggers-Bernard Conference initiative and presents the compiled consensus points. The details of the recommendations/considerations are published in three separate papers [19-21] subsequently appearing in the 2019 January issue of the Shock journal. The Tables 1, 2 and 3 summarize the main MQTiPSS consensus points published in those articles: Part I-Table 1 [19], Part II - Table 2 [20] and Part III-Table 3 [21]. Each publication is built on two (related) Working Group themes and includes a narrative clarifying caveats and intricacies related to the accepted consensus points. 


\section{The future}

The presented consensus has not received formal endorsement from professional bodies. Writing an initial consensus was a strategic decision given that an expert opinion report has a shorter publication turnaround and our intention was to rapidly introduce the MQTiPSS concept. The Wiggers-Bernard Conference was conceived not as a one-time event but rather as an initial "call-to-arms"; an invitation to interested parties to provide further refinement and expansion of the proposed points. The on-going expansion initiatives include formation of a Task Force (under the auspices of the Shock Society; June 2017) for creation of robust, defined parameters to score sepsis models for clinical relevance. Another iteration of the Wiggers-Bernard Conference on animal sepsis models is planned for October 2019 at the joint conference of the European Shock Society and International Federation of Shock Societies in Crete, Greece.

In summary, we believe that the proposed guidelines represent the first concrete steps toward creation of a realistic framework for standardization of animal models of sepsis (i.e. MQTiPSS). Such a framework, once widely employed, will improve the quality of pre-clinical investigation and arm clinicians with better tools for combating sepsis in patients.

Acknowledgements The authors would like to thank Bettina Standhartinger for her valuable assistance in organizing the Wiggers-Bernard Conference.

\section{Compliance with ethical standards}

Conflict of interest Claes Frostell controls the Claes Frostell Research \& Consulting $\mathrm{AB}$ company that participated in supporting several clinical and experimental studies. Other authors do not declare any conflict of interest.

Open Access This article is distributed under the terms of the Creative Commons Attribution 4.0 International License (http://creativeco mmons.org/licenses/by/4.0/), which permits unrestricted use, distribution, and reproduction in any medium, provided you give appropriate credit to the original author(s) and the source, provide a link to the Creative Commons license, and indicate if changes were made.

\section{References}

1. Lamberg L. Researchers urged to tell public how animal studies benefit human health. JAMA. 1999;282:619-21.

2. Van der Worp HB, Howells DW, Sena ES, Porritt MJ, Rewell S, O'Collins V, Macleod MR. Can animal models of disease reliably inform human studies? PLoS Med. 2010;7:e1000245.

3. Stortz JA, Raymond SL, Mira JC, Moldawer LL, Mohr AM, Efron PA. Murine models of sepsis and trauma: can we bridge the gap? ILAR. 2017; J 58:90-105.

4. Van der Poll T. Preclinical sepsis models. Surg Infect (Larchmt). 2012;13:287-92.
5. Coen D. Oxford TB vaccine study calls into question selective use of animal data. BMJ. 2012;360:j5845.

6. Wieschowski S, Chin WWL, Federico C, Sievers S, Kimmelman J, Strech D. Preclinical efficacy studies in investigator brochures: do they enable risk-benefit assessment? PLoS Biol. 2018;16:e2004879.

7. Marshall JC. From the bedside back to the bench: the role of preclinical studies in understanding clinical therapies. Crit Care Med. 2010;38:329-30.

8. Efron PA, Mohr AM, Moore FA, Moldawer LL. The future of murine sepsis and trauma research models. J Leukoc Biol. 2015;98:945-52.

9. Singer M, Deutschman CS, Seymour CW, Shankar-Hari M, Annane D, Bauer M, Bellomo R, Bernard GR, Chiche JD, Coopersmith $\mathrm{CM}$, et al. The third international consensus definitions for sepsis and septic shock (Sepsis-3). JAMA. 2016;315:801-10.

10. Rhodes A, Evans LE, Alhazzani W, Levy MM, Antonelli M, Ferrer R, Kumar A, Sevransky JE, Sprung CL, Nunnally ME, et al. Surviving sepsis campaign: international guidelines for management of sepsis and septic shock: 2016. Crit Care Med. 2017;45:486-552.

11. Remick DG, Ayala A, Chaudry I, Coopersmith CM, Deutschman C, Hellman J, Moldawer L, Osuchowski M. Premise for standardized sepsis models. Shock. 2018. https://doi.org/10.1097/ SHK.0000000000001164.

12. Jenkins RG, Moore BB, Chambers RC, Eickelberg O, Königshoff M, Kolb M, Laurent GJ, Nanthakumar CB, Olman MA, Pardo A, et al. ATS assembly on respiratory cell and molecular biology. An Official American Thoracic Society Workshop Report: use of animal models for the preclinical assessment of potential therapies for pulmonary fibrosis. Am J Respir Cell Mol Biol. 2017;56:667-79.

13. Albers GW, Anwer UE, Ashwood T, Barone FC, Basta SL, Bogousslavsky J, Buchan AM, Cady WJ, Chan PH, Clemens JA, et al. Recommendations for standards regarding preclinical neuroprotective and restorative drug development. Stroke Therapy Academic Industry Roundtable (STAIR). Stroke. 1999;30:2752-58.

14. Vahidy F, Schäbitz WR, Fisher M, Aronowski J. Reporting standards for preclinical studies of stroke therapy. Stroke. 2016;47:2435-38.

15. Houser SR, Margulies KB, Murphy AM, Spinale FG, Francis GS, Prabhu SD, Rockman HA, Kass DA, Molkentin JD, Sussman MA, et al. American Heart Association Council on Basic Cardiovascular Sciences, Council on Clinical Cardiology, and Council on Functional Genomics and Translational Biology. Animal models of heart failure: a scientific statement from the American Heart Association. Circ Res. 2012;111:131-50.

16. Craig AG, Grau GE, Janse C, Kazura JW, Milner D, Barnwell JW, Turner G. Langhorne J and participants of the Hinxton Retreat meeting on Animal Models for Research on Severe Malaria. The role of animal models for research on severe malaria. PLoS Pathog. 2012;8:e1002401.

17. Osuchowski MF, Thiemermann C, Remick DG. Sepsis-3 on the block: what does it mean for preclinical sepsis. Modeling? Shock. 2017;47:658-60.

18. Marshall JC, Deitch E, Moldawer LL, Opal S, Redl H, van der Poll T. Preclinical models of shock and sepsis: what can they tell us? Shock. 2005;24(Suppl 1):1-6.

19. Zingarelli B, Coopersmith CM, Drechsler S, Efron P, Marshall JC, Moldawer LL, Wiersinga WJ, Xiao X, Osuchowski MF, Thiemermann C, et al. Part I: Minimum quality threshold in pre-clinical sepsis studies (MQTiPSS) for study design and humane modeling endpoints. Shock. 2019;51(1):4-17.

20. Libert C, Ayala A, Bauer M, Cavaillon J-M, Deutschman C, Frostell C, Knapp S, Kozlov AV, Wang P, Osuchowski MF, et al. Part II: Minimum quality threshold in pre-clinical sepsis studies 
(MQTiPSS) for types of infections and organ dysfunction endpoints. Shock. 2019;51(1):18-27.

21. Hellman J, Bahrami S, Boros M, Chaudry I, Fritsch G, Gozdzik W, Inoue S, Radermacher P, Singer M, Osuchowski MF, et al.
(2018) Part III: minimum quality threshold in pre-clinical sepsis studies (MQTiPSS) for fluid resuscitation and antimicrobial therapy endpoints. Shock. 2019;51(1):28-38. https://doi.org/10.1097/ SHK.0000000000001209.

\section{Affiliations}

\section{Marcin F. Osuchowski ${ }^{1}$. Alfred Ayala ${ }^{2}$. Soheyl Bahrami ${ }^{1} \cdot$ Michael Bauer $^{3} \cdot$ Mihaly Boros $^{4} \cdot$ Jean-Marc Cavaillon ${ }^{5}$. Irshad H. Chaudry ${ }^{6}$. Craig M. Coopersmith ${ }^{7}$. Clifford Deutschman ${ }^{8}$. Susanne Drechsler ${ }^{1}$. Philip Efron ${ }^{9}$. Claes Frostell ${ }^{10}$. Gerhard Fritsch ${ }^{11,12}$. Waldemar Gozdzik ${ }^{13}$. Judith Hellman ${ }^{14}$. Markus Huber-Lang ${ }^{15}$. Shigeaki Inoue ${ }^{16}$. Sylvia Knapp ${ }^{17}$. Andrey V. Kozlov ${ }^{1}$. Claude Libert ${ }^{18,19}$. John C. Marshall ${ }^{20}$. Lyle L. Moldawer ${ }^{9}$. Peter Radermacher ${ }^{21}$ • Heinz Redl ${ }^{1}$ - Daniel G. Remick ${ }^{22} \cdot$ Mervyn Singer $^{23} \cdot$ Christoph Thiemermann $^{24} \cdot$ Ping Wang $^{25}$. W. Joost Wiersinga ${ }^{26} \cdot$ Xianzhong Xiao $^{27} \cdot$ Basilia Zingarelli $^{28}$}

1 Ludwig Boltzmann Institute for Experimental and Clinical Traumatology in the AUVA Research Center, Donaueschingenstrasse 13, 1200 Vienna, Austria

2 Rhode Island Hospital and Alpert School of Medicine at Brown University, Providence, RI, USA

3 Jena University Hospital, Jena, Germany

4 Institute of Surgical Research, University of Szeged, Szeged, Hungary

5 Institut Pasteur, Paris, France

6 University of Alabama at Birmingham School of Medicine, Birmingham, AL, USA

7 Emory University School of Medicine, Atlanta, GA, USA

8 Feinstein Institute for Medical Research, Northwell Health, Manhasset, NY, USA

9 University of Florida College of Medicine, Gainesville, FL, USA

10 Division of Anaesthesia and Intensive Care, Karolinska Institutet, Danderyd Hospital, Stockholm, Sweden

11 AUVA Traumacenter, Vienna, Austria

12 Paracelsus Medical University, Salzburg, Austria

13 Wroclaw Medical University, Wroclaw, Poland

14 School of Medicine, University of California, San Francisco, San Francisco, CA, USA

15 Institute of Clinical and Experimental Trauma-Immunology, University Hospital of Ulm, Ulm, Germany

16 Kobe University Graduate School of Medicine, Kobe, Japan
17 Department of Medicine 1, Medical University Vienna, Vienna, Austria

18 Center for Inflammation Research, VIB, Ghent, Belgium

19 University Ghent, Ghent, Belgium

20 Keenan Research Centre for Biomedical Science, St. Michael's Hospital, University of Toronto, Toronto, Canada

21 Institute of Anaesthesiological Pathophysiology and Process Development, University Hospital of Ulm, Ulm, Germany

22 Boston University School of Medicine, Boston, MA, USA

23 Bloomsbury Institute of Intensive Care Medicine, University College London, London, UK

24 The William Harvey Research Institute, Barts and London School of Medicine and Dentistry, Queen Mary University of London, London, UK

25 Feinstein Institute for Medical Research, Manhasset, NY, USA

26 Division of Infectious Diseases, and Center for Experimental and Molecular Medicine, the Academic Medical Center, University of Amsterdam, Amsterdam, The Netherlands

27 Xiangya School of Medicine, Central South University, Chagnsha, Hunan, China

28 Division of Critical Care Medicine, Cincinnati Children's Hospital Medical Center, College of Medicine, University of Cincinnati, Cincinnati, OH, USA 